\title{
Metastatic malignant melanoma: a case report
}

\begin{abstract}
Metastatic malignant melanoma is a major cause of premature death from cancer. ${ }^{1}$ Survival of melanoma has increased to greater than $90 \%$ due to early detection and screening. 1 However there are several atypical melanomas which are hard to recognize, namely nevoid and amelanotic melanoma, which can have bad prognosis. ${ }^{2}$ Sentine ${ }^{1}$ lymph node status remains an important prognostic factor for survival of patients with melanoma. ${ }^{1}$ Research has shown that exposure to UV radiation increases the melanoma risk. ${ }^{3}$ Functional mutation in the mitogen activated pathways are common in melanomas and this influence the growth control. ${ }^{1}$ About $10 \%$ of the melanoma patients report a family history of melanoma. ${ }^{4}$ CDKN2A mutations remains the most common one. ${ }^{4}$ Several studies has made suggestions for the use of dermoscopy and total body photography for the screening. ${ }^{4}$ Management of melanomas usually involve several components such as excision, tumor staging, reexcision for negative margins, adjuvant therapies (chemo, radiation or surgery), follow-up examination for metastasis, lifestyle modification and counseling. ${ }^{1}$ Metastatic malignant melanoma has very poor prognosis however development of targeted immunotherapy has changed the outcomes in this patient population. ${ }^{1}$ We are presenting a case of a 43 year old female with metastatic malignant melanoma with a very poor prognosis due to her extensive metastasis.
\end{abstract}

Keywords: malignant melanoma, tumor staging, re-excision, distant metastasis, locoregional lymph
Volume 10 Issue 6 - 2019

\author{
Chintav Shah,' Jay Patolia,' Simeon \\ Jaggernauth, ${ }^{2}$ Jason Kimbel' \\ 'Department of Internal Medicine, University of Arkansas for \\ Medical Sciences, USA \\ ${ }^{2}$ Landmark Cancer Center, Arkansas, USA
}

Correspondence: Chintav Shah, Department of Internal Medicine, University of Arkansas for Medical Sciences, Fayetteville, Arkansas, USA, Email Chintavshah6@gmail.com, chsha@uams.edu

Received: December 16, 2019 | Published: December 23, 2019

\section{Background}

The incidence of melanoma has been increasing although the cancer of the other sites is decreasing. ${ }^{3}$ Malignant melanoma are derived from the melanocytes.1Skin is the most common site of occurrence however other organs like eye, oral and nasal mucosa, anorectal mucosa and CNS tend to occur. ${ }^{1}$ It is the 5 th most common cancer in men. ${ }^{5}$ The 5 -year survival rate is only $15 \%$ for distant metastasis, improved only slightly from $12 \%$ in the past decade. ${ }^{5} 70 \%$ of all cutaneous melanomas have CDKN2A mutations. ${ }^{5}$ Mutations in $\mathrm{N}-\mathrm{RAS}$ and BRAF, the proteins involved in MAPK pathway were found in $20 \%$ and $60 \%$ cases respectively. ${ }^{5}$ The most commonly seen BRAF mutation is a substitution of valine with glutamate (V600E). ${ }^{5}$ The most common sites of melanoma are legs in women and back in men. ${ }^{1}$ Macroscopic locoregional lymph node metastasis reduces fiveyear survival to $43 \%$. Patients who had locoregional metastasis had a better survival rate than those with metastasis to viscera. ${ }^{1}$ Sentinel lymph node status remains an important prognostic factor for survival of patients with melanoma. ${ }^{1}$

\section{Case report}

This is a 43yr old female with a history of malignant melanoma involving the right shoulder following resection approximately $10 y e a r s$ ago without axillary node sampling. She presented to her PCP with complaints of nausea, vomiting and right shoulder pain. In the process of her evaluation, she had an ultrasound of the abdomen which showed an ill-defined hypoechoic focus on right hepatic lobe measuring $1.4 \times 1.6 \times 1.3 \mathrm{~cm}$. A solid appearing exophytic mass in the lower pole of the right kidney was also seen measuring $1.0 \times 1.2 \times 1.4 \mathrm{~cm}$. Given these findings, a CT scan of the abdomen was performed which showed extensive nodular peritoneal and retroperitoneal implants throughout the abdomen and anterior to the right kidney. Omental caking was also noted with multiple small nodules seen with the largest measuring $8 \mathrm{~mm}$. Metastatic disease was seen within the liver and L5. Subcutaneous lesions were also seen in the breasts bilaterally which were biopsy confirmed.

A repeated CT was done a month later which showed above findings along with several hypodense lesions in the spleen thought to represent splenic metastasis. Patient presented to cancer clinic for further evaluation. In the interim she had an MRI of the brain showing no evidence of malignancy. She underwent biopsy of the left breast which was found to be consistent with malignant melanoma. BRAF analysis was negative. A PET scan was performed which showed uptake within bilateral cervical and supraclavicular as well as right axillary and subpectoral lymph nodes. Multiple breast nodules were seen with SUV of 6.0. Within abdomen pelvis was subtle lesion on the right hepatic lobe with SUV of 1.9 with multiple avid nodes in the abdomen and retroperitoneum with SUV of up to 2.6. Uptake was noted within the left posterior thigh with a lesion measuring 0.6 $\mathrm{cm}$ with SUV of 3.0. There were widespread lesions throughout the skeleton with SUV up to 4.0. She had a very poor prognosis with an expected survival of 6 to 8 weeks.

She was started on combination immunotherapy with Opdivo and Yervoy. She completed 4 cycles of it and had a repeat PET. She had resolution of the multiple breast lesions bilaterally as well as interval resolution of multiple FDG avid lesions in the abdomen and retroperitoneum. No activity was identified in the liver. She also had resolution of the FDG avid lesions in the thigh and the foot. Interval resolution of the widespread FDG avid lesions was also noted which 
were now sclerotic in nature. Overall she had an excellent response to therapy.

She was continued on the maintenance opdivo but then developed some pain in the tail bone. She had a positive PET scan in this region therefore she underwent radiation therapy. The PET scan also demonstrated activity alone in the left breast and she underwent a mastectomy with pathology showing evidence of a foreign giant cell reaction with fat necrosis and no residual evidence of melanoma was identified.

She was continued on maintenance immunotherapy for 18 cycles. She had a repeat PET scan exactly a year later from her first one. This PET showed no evidence of any FDG avid lesions. Minimal activity involving the anterior lymph nodes was seen which was thought to be benign.

She experienced some minor complications from pneumonitis thought to be secondary to her immunotherapy which was then held. She was treated with steroids resulting in resolution. She was started back on the maintenance opdivo and she has remained on it since initiation. Three subsequent PET scans have remained negative for any detectable metastatic disease and she remained in complete remission.

\section{Discussion}

Metastatic malignant melanoma has a very grave prognosis with 5 year survival of $15 \% .^{5}$ The US Preventive Services Task Force (USPSTF) recommends neither for nor against screening by the PCP for an early diagnosis. ${ }^{6}$ With the identification of the genes and susceptibility, has led to the emergence of several small molecule inhibitors. ${ }^{5}$ A multicenter phase 3 trial suggested that the BRAF inhibitor, vemurafenib, might extend survival in patients with advanced disease harboring the V600E mutation. ${ }^{5}$ Trametinib, inhibitor of MEK1 and MEK2 which are downstream of BRAF in MAPK pathway was also shown to have comparable efficacy as with Vemurafinib. ${ }^{5}$ Melanoma cells evade immunologic clearance.5The approval of the immunological checkpoint regulators has been very important as demonstrated in this patient. Ipilimumab became the first FDA-approved antibody-based immunotherapy for melanoma. ${ }^{5}$ By binding to CTLA 4, Ipilimumab potentiates antitumor immunity and has shown to increase survival in patients with metastatic malignant melanoma who has refractory disease. ${ }^{5}$ Another agent that is approved for the treatment called nivolumab directed against PD-1, an inhibitory receptor on activated T-cells and its ligand. ${ }^{5}$ Despite these numerous advances, the response to the above therapy can vary and has not been consistent. However, as in the above case, the patient had an excellent response to immunotherapy therapy resulting in a complete remission clinically, pathologically, and radio graphically.

\section{Acknowledgments}

None.

\section{Conflicts of interest}

The authors declare there is no conflict of interests regarding the publication of this paper.

\section{References}

1. Perera E, Gnaneswaran N, Jennens R, et al. Malignant melanoma. Healthcare (Basel). 2013;2(1):1-19.

2. Cabrera R, Recule F. Unusual clinical presentations of malignant melanoma: a review of clinical and histologic features with special emphasis on dermatoscopic findings. Am J Clin Dermatol.2018;19(Suppl 1): $15-23$.

3. Tripp MK, Watson M, Balk SJ, et al. State of the science on prevention and screening to reduce melanoma incidence and mortality: the time is now. CA Cancer J Clin. 2016;66(6):460-480.

4. Soura E, Eliades PJ, Shannon K, et al. Hereditary melanoma: update on syndromes and management - genetics of familial atypical multiple mole melanoma syndrome. J Am Acad Dermatol. 2016;74(3):395-407.

5. Lee N, Barthel SR, Schatton T. Melanoma stem cells and metastasis: mimicking hematopoietic cell trafficking? Lab Invest. 2014;94(1):1330 .

6. Harrington E, Clyne B, Wesseling N, et al. Diagnosing malignant melanoma in ambulatory care: a systematic review of clinical prediction rules. BMJ Open. 2017;7(3):e014096. 